\title{
Influence of vortex domain walls on magnetoresistance signals in Permalloy rings
}

\author{
Mei-Feng Lai, ${ }^{1}$ Zung-Hang Wei, ${ }^{1, *}$ Ching-Ray Chang, ${ }^{1}$ J. C. Wu, ${ }^{2}$ J. H. Kuo, ${ }^{2}$ and Jun-Yang Lai ${ }^{1}$ \\ ${ }^{1}$ Department of Physics, National Taiwan University, Taipei, Taiwan, 10617, Republic of China \\ ${ }^{2}$ Department of Physics, National Changhua University of Education, \\ Changhua, Taiwan, 500, Republic of China
}

(Received 28 July 2002; revised manuscript received 17 December 2002; published 20 March 2003)

\begin{abstract}
The magnetization reversal and the corresponding magnetoresistance of Permalloy rings are investigated here both numerically and experimentally. Micromagnetic simulations reveal that during the reversal process there exist three intermediate metastable states which lead to an unconventional triple-switching characteristic. Size effects are studied as well. The simulation results are in good agreement with our magnetoresistance measurements. Significant differences in the magnetoresistance at two orthogonal directions of the magnetic fields are observed and explained. An important contribution to the magnetoresistance due to the relative position of the domains and the current leads is also investigated.
\end{abstract}

DOI: 10.1103/PhysRevB.67.104419

PACS number(s): 75.60.Jk, 75.75.+a

\section{INTRODUCTION}

The rapid development of magnetic data storage and magnetoelectronic devices technology ${ }^{1}$ has prompted the study of magnetization reversal on submicron ferromagnetic elements. Ferromagnetic patterned thin films differ from continuous thin films both in their spin configurations and in their reversal properties due to the presence of boundaries. ${ }^{2}$ In patterned Permalloy thin films the magnetization normal to the boundaries causes the accumulation of magnetic pole densities which increase the magnetostatic energy. Among various patterned elements, the ring structure has become a promising candidate for the magnetic random access memory (MRAM). ${ }^{3}$ Due to its circular shape it easily forms stable flux-closure states whose existence impedes field leakage and reduces the influence of edge domains. The data storage density of the devices and the uniformity of the switching process ${ }^{3}$ are thus enhanced. However, lack of repeatability and of uniform performance is still a serious problem during accessing the MRAM cell. It is therefore imperative to understand the relationship between the domain structure of the ring, its reversal process, and the magnetoresistance (MR) properties.

In this paper we investigate, both theoretically and experimentally, the magnetization reversal and the magnetoresistance of small Permalloy rings. We clarify the reversal dynamics, and discuss in some detail the influence which domains short circuited by contact leads have on the magnetoresistance of the rings.

\section{SIMULATION DETAILS}

In our three-dimensional numerical simulation the thickness of the ring element is $30 \mathrm{~nm}$, and the outer and inner diameters are 375 and $225 \mathrm{~nm}$, respectively. The micromagnetic properties of these submicron-sized ring elements are investigated numerically by the time integration of the Landau-Lifshitz-Gilbert (LLG) equation; ${ }^{4,5}$ with exchange interactions, magnetostatic interactions, anisotropies, and external fields all taken into account. The material parameters are chosen to agree with soft Permalloy films, so that we set the exchange constant $C=2 A=2 \times 10^{-6} \mathrm{erg} / \mathrm{cm}$, the saturation magnetization $M_{s}=800 \mathrm{emu} / \mathrm{cm}^{3}$, and the uniaxial anisotropy constant $K_{1}=10^{3} \mathrm{erg} / \mathrm{cm}^{3}$. In the simulation we divide the ring element into small cubic cells approximating the ring shape. The length of each of these mesh cells is 7.5 $\mathrm{nm}$, i.e., less than half of the exchange length of the Permalloy material, ${ }^{6} R_{0}=\sqrt{C} / M_{s}=17.7 \mathrm{~nm}$, and therefore satisfies the usual requirement that it be much less than the exchange length. Our simulation is based on an iterative method in which the alignment between the magnetization and its local effective field is successively adjusted. The simulation terminates when an equilibrium state is reached. The iterations proceed in the following steps: First we use the initial spin configuration to calculate the effective field at all cubic cells. Then the magnetization of each cubic cell is simultaneously adjusted in the direction of its effective field according to the LLG equation. The damping constant is set to be 1 to assure rapid convergence. In the next iteration, the spin configuration just obtained is used to update the effective field in all cells, and a new, updated spin configuration is then computed. It is assumed that the equilibrium configuration has been reached if the largest angular variation of magnetization in each cell is below the value $10^{-8}$ in two successive iterations. Finally we test the stability of the equibrium state by applying a small perturbation to the computed spin configuration. If the configuration is stable then the initial perturbation steadily decreases in successive iterations.

\section{STABLE STATES AND MAGNETIZATION REVERSAL}

Figure 1 shows our simulation results for three different spin configurations of the Permalloy ring. The magnitudes and directions of the external applied field are indicated by arrows inside the rings. The schematics are also plotted in the figure to symbolize the spin configurations. The magnetization of the ring is first saturated by a large field of 3000 $\mathrm{Oe}$, and the field is then reduced to zero. The resultant remanent spin configuration is shown in Fig. 1(a). The net magnetization of the ring remains in the direction of the original applied field, but the magnetization becomes locally aligned with the ring edges. We call this a quasiuniform state. There are two transverse walls ${ }^{7}$ at the two ends of the ring. On a further decrease of the field from 0 to -145 Oe, there occurs 

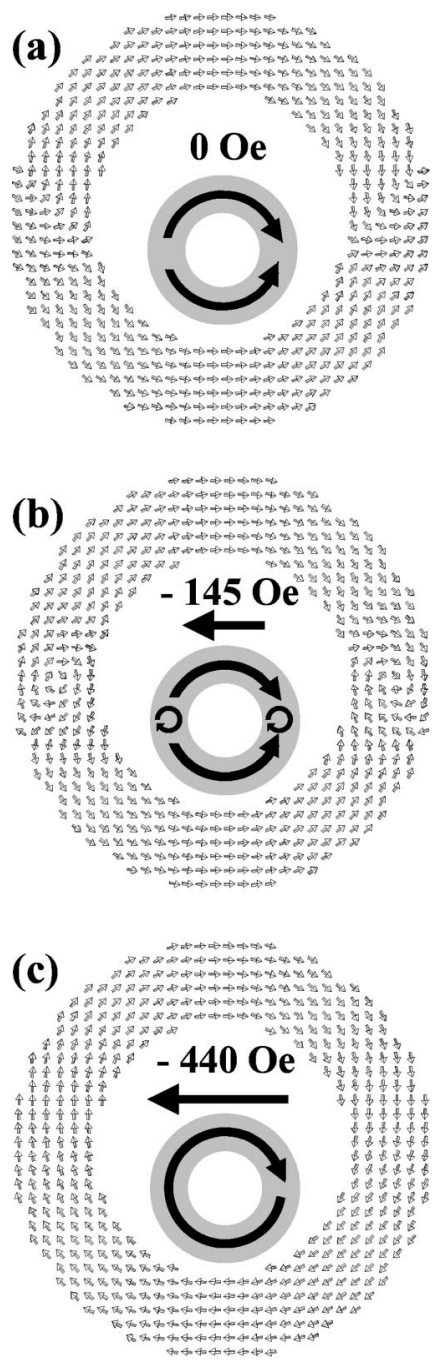

FIG. 1. Typical simulated spin configurations for the Permalloy ring. The external field and the schematics are indicated in the figure. The ring is $30 \mathrm{~nm}$ thick, and has an outer diameter of $375 \mathrm{~nm}$ and an inner diameter of $225 \mathrm{~nm}$. For better visual clarity the computed vectors are represented by arrows only at selected points of the ring.

the first transition in which two small vortices are nucleated at the outer edge of the ring near the transverse domain walls. The spin configuration now changes from the quasiuniform state to a vortex-pair state, which resembles the quasiuniform state except for the existence of the two small vortices, as shown in Fig. 1(b). The presence of the negative external and the demagnetization fields makes it impossible to maintain the quasiuniform structure, and the nucleation of the two vortices leads to a reduction of the magnetostatic energy. A second transition occurs if the field is further decreased to -440 Oe: The two small vortices now move to the lower half of the ring, approach each other, and are finally annihilated. The spin configuration changes from the vortexpair state to the flux-closure state shown in Fig. 1(c). In this configuration the spins of the ring essentially align to form a closed loop, and the net magnetization is almost zero. This transition causes an abrupt drop in the total magnetization along the applied field direction. The third transition occurs

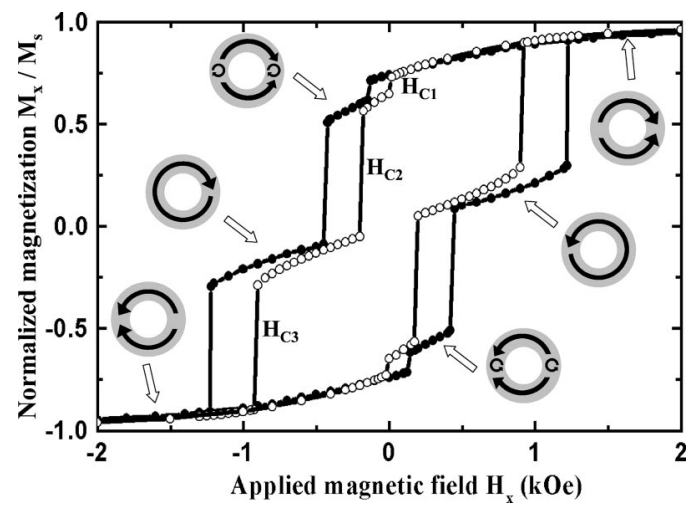

FIG. 2. Simulated hysteresis loops for Permalloy rings of different sizes. The black line with solid circles corresponds to a ring with a thickness of $30 \mathrm{~nm}$, an outer diameter of $375 \mathrm{~nm}$, and an inner diameter of $225 \mathrm{~nm}$. The black line with open circles corresponds to a ring with a thickness of $30 \mathrm{~nm}$, an outer diameter of 600 $\mathrm{nm}$, and an inner diameter of $360 \mathrm{~nm}$. The schematic figures show the stable spin configurations at the plateaus. The switching fields between the four stable states are $H_{C 1}, H_{C 2}$, and $H_{C 3}$.

when the field is further decreased to -1225 Oe. The upper half of the ring undergoes a nonuniform reversal, but at the same time the spin configuration of the lower half remains almost unchanged. A nucleation at the inner edge of the ring takes place during this transition, and the spin configuration changes from flux-closure state to a quasiuniform state magnetized in the reverse direction. The magnetization reversal processes just described for the second and third transitions are similar to the reversal of an asymmetric cobalt ring. ${ }^{8}$ The asymmetry in our simulation is believed to come from edge roughness which leads to a slight variation of the switching field. ${ }^{8}$

Figure 2 shows simulated hysteresis loops of two Permalloy rings of different sizes. The two rings have the same thickness and the same aspect ratio which we define as the ratio between the outer and inner diameters. The transitions in the two corresponding hysteresis loops have similar features. In the sweep-down process each of the hysteresis loops exhibits an unconventional triple-switching behavior in which the magnetization reversal occurs successively at three different fields: $H_{C 1}, H_{C 2}$, and $H_{C 3}$, as shown in Fig. 2. The resultant four plateaus correspond to the quasiuniform state, the vortex-pair state, the flux-closure state, and the reverse quasiuniform state, respectively. The same features are observed on the reverse sweep of the field. We note that the three switching fields $H_{C 1}, H_{C 2}$, and $H_{C 3}$ shift to the right as the ring size increases. In the larger ring two small vortices naturally form in the opposite ends at remanence ${ }^{7}$, even at positive field (see Fig. 2). This vortex structure decreases the demagnetization energy of the wider ring. There is, however, a smaller increase in the exchange energy, but the total energy is lowered. Figure 2 also shows that the overall coercivity is decreased in larger rings.

\section{SAMPLE FABRICATION}

We fabricated the ring-shaped Permalloy thin film devices by a two-step standard electron beam lithography and ther- 
(a)

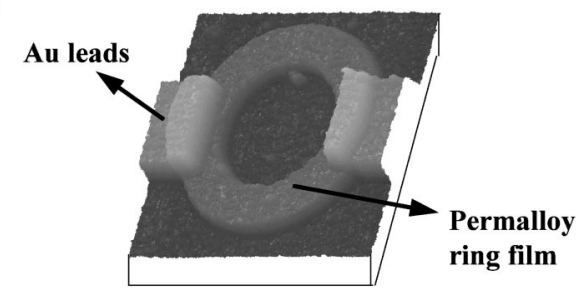

(b)
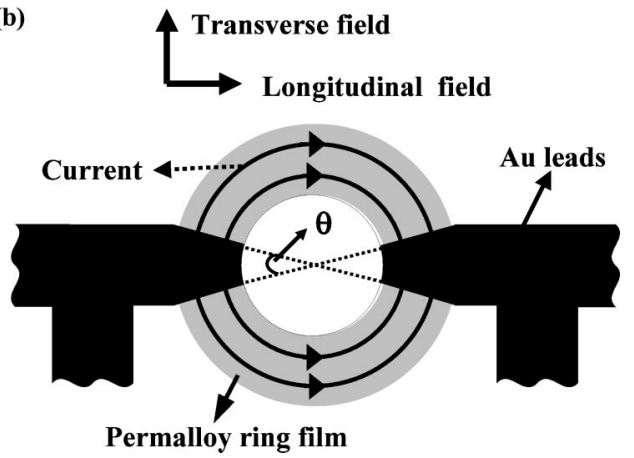

FIG. 3. (a) An AFM picture of the ring sample. The outer diameter, the inner diameter, and the thickness are $5 \mu \mathrm{m}, 3 \mu \mathrm{m}$, and 450 $\AA$, respectively. (b) Schematic picture of the simulation model. The outer diameter, the inner diameter, and the thickness are $375 \mathrm{~nm}$, $225 \mathrm{~nm}$, and $30 \mathrm{~nm}$, respectively.

mal evaporation through a lift-off technique. First, ring patterns of diameter $5 \mu \mathrm{m}$ and line width $1 \mu \mathrm{m}$ are exposed in electron beam resist, polymethyl methacrylate (PMMA), using a commercial scanning electron microscope modified for direct writing. After the sample is developed in a 1:3 mixture of methyl isobutyl ketone and 2-propanol, the ring-shaped trenches are created in PMMA. A thermal evaporation system is used for the Permalloy deposition in which a thickness of $450 \AA$ is monitored by a quartz crystal. During the Permalloy deposition the substrate temperature is kept at room temperature and no external magnetic field is present. The polycrystalline Permalloy rings are then transferred onto a SiN-coated silicon substrate after a lift-off process in acetone. In the next stage one more step of electron beam lithography is carried out to fashion current/voltage leads of nonmagnetic material; usually $10 \mathrm{~nm}$ of Ti followed by 50 $\mathrm{nm}$ of $\mathrm{Au}$ are used, and the linewidth is $1 \mu \mathrm{m}$. This second lithographic step is adopted to avoid formation of domain walls at the intersection due to shape discontinuities.

\section{MR MEASUREMENT AND THEORETICAL MODEL}

Room temperature magnetoresistance measurements are carried out with 1-mA dc sensing current and under an external magnetic field applied in the film plane longitudinal or transverse to the current between electrodes. Figure 3(a) shows an atomic force microscopy (AFM) picture of the sample.

Figure 3(b) shows the schematic plot of the model used in our theoretical calculation following the setup of the experimental device. The electrodes of $1-\mu \mathrm{m}$ width are patched onto the Permalloy ring at an angle $\theta \approx 22.5^{\circ}$, which is also adopted in our calculation model. The magnetic field is ap- plied in transverse and longitudinal directions as shown in the figure. The current density vector $\vec{J}$ in our calculation is assumed to be uniform, and to flow along the circumference as shown in Fig. 3(b).

The magnetoresistance of ferromagnetic materials is usually affected by temperature and external field, and contains contributions from the domains and the domain walls. ${ }^{9}$ The contribution of very narrow domain walls (within a few nanometers), which are usually due to high anisotropy ${ }^{10}$ or nanoconstrictions, ${ }^{11,12}$ is often important. In permalloy thin films the anisotropy is very small, so the domain wall is wider and the domain wall scattering can therefore be neglected. In ferromagnetic materials the resistance anisotropy has two competing terms, the anisotropic magnetoresistance (AMR) and the Lorentz MR, and they are of opposite signs. ${ }^{9}$ At low temperatures the Lorentz MR becomes important, while at high temperatures the AMR is dominant. ${ }^{9}$ Our experiment is done at room temperature where the Lorentz MR can be neglected. The AMR effect is due to anisotropic spinorbit interactions which give rise to a resistance determined by the relative orientation of the current density and the magnetization. ${ }^{13}$ The anisotropic magnetoresistance of the Permalloy ring is given by the equation ${ }^{14}$

$$
\rho_{A}(\vec{M}, \vec{J} ; \alpha)=\rho_{\perp}\left(1+\frac{\Delta \rho}{\rho_{\perp}} \cos ^{2} \alpha\right),
$$

where $\alpha$ is the angle spanned by the magnetization vector $\vec{M}$ and the current density vector $\vec{J}$. In the difference $\Delta \rho=\rho_{\|}$ $-\rho_{\perp}$ the term $\rho_{\perp}$ is the resistivity at $\alpha=90^{\circ}$, and $\rho_{\|}$is the resistivity at $\alpha=0^{\circ}$. Because our experiment is done at room temperature, we set $\Delta \rho / \rho_{\perp}=0.02$ for NiFe Permalloy in accordance with the experimental work of Adeyeye et al., ${ }^{15}$ and with the theoretical work by Rijks et al. ${ }^{16}$ Finally, we use the magnetization $\vec{M}$ derived from our simulation to obtain the magnetoresistance.

\section{RESULTS AND DISCUSSION}

First we consider the case of a Permalloy ring subject to a transverse field. Figures 4(a) and 4(b) represent the transverse MR curves obtained by simulation and experiment, respectively. The quasiuniform state, the vortex-pair state, the flux-closure state, and the reverse quasiuniform state (which correspond to the external field regions I, II, III, and IV as the field sweeps down) are schematically represented in the lower parts of figures. These spin configurations are similar to those shown in Figs. 1(a)-1(c). The spatial relationship between the domains and the leads is shown in the inset of Fig. 4(b). Note that the domain walls are not hidden under the leads. Three transitions between the metastable states I-IV take place during the field sweep: In the transition from the state I to state II two small vortices are formed at the opposite ends of the ring, and this causes a slight decrease in the magnetoresistance as shown in Figs. 4(a) and 4(b). In the next transition from II to III the two small vortices disappear, and the spins form the closed flux loop configuration. During this transition the magnetoresitance increases abruptly since in flux-closure state the magnetization and the current have the same orientation. In the last transi- 


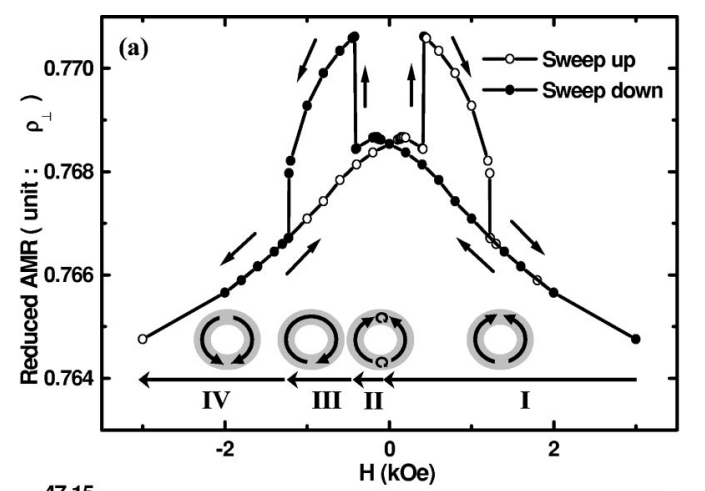

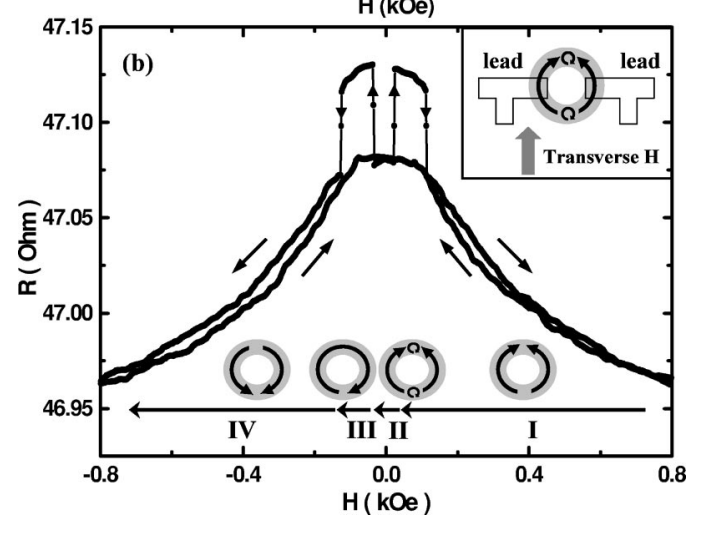

FIG. 4. Transverse MR curves of Permalloy ring obtained by (a) theoretical and (b) experimental methods. The spatial relationship between the domains and leads is shown in the inset. The metastable stable states observed in the sweep-down process are schematically represented in the lower part of each figure.

tion, from state III to state IV, the magnetoresistance decreases considerably due to the nucleation of the two transverse domain walls of the quasiuniform state. The MR curve of final state IV is similar to that of initial state I.

Figures 5(a) and 5(b) represent the longitudinal MR curves obtained, respectively, by simulation and in experiment. The Permalloy ring is in this case subject to a longitudinal field. The four metastable states and their existent domains are schematically represented in the lower parts of the plots, together with the spatial relationship between the domains and the leads. Here the domain walls are partially hidden under the leads; this circumstance contributes to the observed significant difference between the transverse and the longitudinal MR curves. The Au leads are nearly equipotential, so that the current only flows through the contact leads but not through the patched magnetic film below. The magnetic domain walls under the leads are therefore short circuited and make no contribution to the magnetoresistance. The characteristics of the longitudinal MR curves are thus very different from the characteristics of the transverse MR curves. In particular, the anomalous drops of the longitudinal MR curves near the transition points are believed to be due to the short-circuiting of the domain wall structures under the leads. The significantly different MR signals in the transverse and longitudinal directions are very useful in the design of spintronic devices. Note also that the characteristics of the MR signals are dependent on the electrodes' dimensions, especially so in the longitudinal MR case, and the dimensions
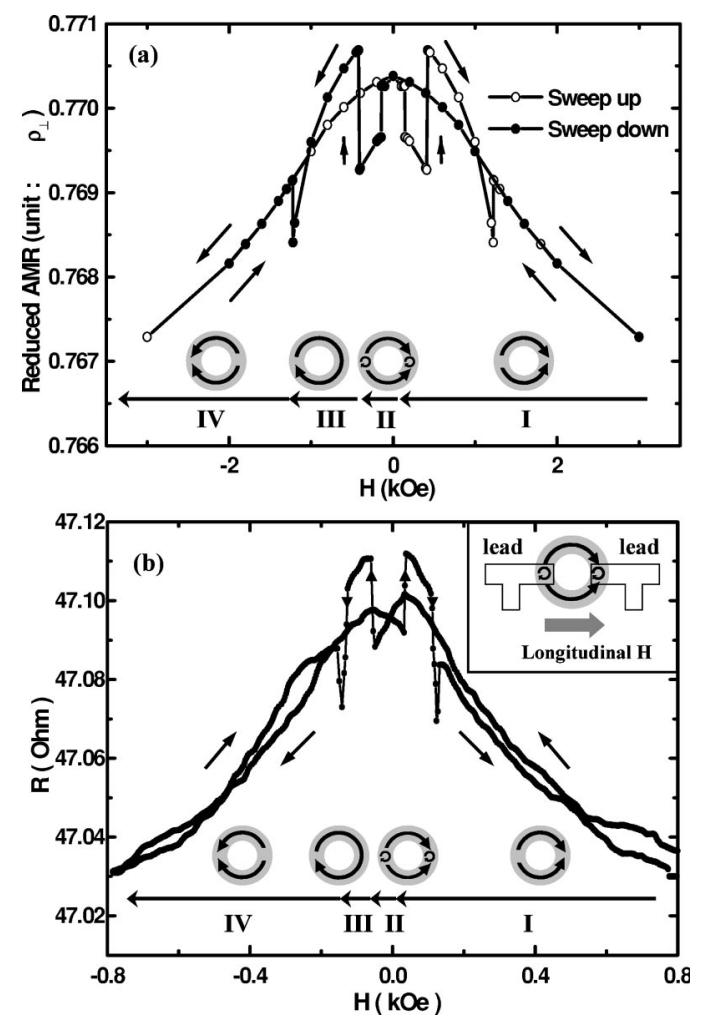

FIG. 5. Longitudinal MR curves of Permalloy ring obtained by (a) theoretical and (b) experimental methods. The spatial relationship between the domains and the leads is shown in the inset. The metastable stable states observed in the sweep-down process are schematically represented in the lower part of each figure.

of the electrodes must therefore be taken into account in the design of experiments. It must be emphasized that in the sweep-down process, when the ring is as small as in our simulation, vortices can only be nucleated under negative external field, while the vortex-pair state is energetically favorable even under positive field in a ring as large as the one used in the experiment. This effect can be interpreted by reference to Fig. 2: In the sweep-down process the three switching fields all shift to the right as the ring size increases, and this explains why the first transition occurs at -145 Oe in simulation (smaller ring size) and at 50 Oe in experiment (larger ring size). Figure 2 also shows that the overall coercivity decreases in larger rings, and this is the reason why the experimental switching fields are much smaller than the computed ones.

\section{CONCLUSIONS}

In summary, we have investigated the magnetization reversal and the magnetoresistance of submicron and micron sized Permalloy rings experimentally and by numerical simulations. Our simulation results are in very good agreement with the experiments, showing that Permalloy rings of micron and submicron sizes have similar switching characteristics. The observed dissimilarities between theoretical and experimental MR curves are due to the dependence of the spin configuration on the dimension of the ring. 
In addition to determining the reversal characteristics of the Permalloy ring our work also clarifies the influence of contact leads on the magnetoresistance response. Magnetic domain walls hidden under the leads, in particular, have a significant influence on the uniformity and repeatability of measurements.

*Electronic address: zhwei@phys.ntu.edu.tw

${ }^{1}$ G. A. Prinz, Science 282, 1660 (1998).

${ }^{2}$ Thomas Schrefl, Josef Fidler, K. J. Kirk, and J. N. Chapman, J. Magn. Magn. Mater. 175, 193 (1997).

${ }^{3}$ Jian-Gang Zhu and Youfeng Zheng, J. Appl. Phys. 87, 6668 (2000).

${ }^{4}$ M. E. Schabes, J. Magn. Magn. Mater. 95, 249 (1991).

${ }^{5}$ N. A. Usov and S. E. Peschany, J. Magn. Magn. Mater. 130, 275 (1994).

${ }^{6}$ Eric D. Boerner, H. Neal Bertram, and Harry Suhl, J. Appl. Phys. 87, 5389 (2000).

${ }^{7}$ R. D. McMichael and M. J. Donahue, IEEE Trans. Magn. 33, 4167 (1997).

${ }^{8}$ J. Rothman, M. Klaui, L. Lopez-Diaz, C. A. F. Vaz, A. Bleloch, J. A. C. Bland, Z. Cui, and R. Speaks, Phys. Rev. Lett. 86, 1098 (2001).

\section{ACKNOWLEDGMENTS}

This work was supported partly by the Republic of China National Science Council Grant No. NSC 91-2119-M002001 and Technology Development Program for Academia Grant No. 91-EC-17-A-08-S1-0006.

${ }^{9}$ U. Ruediger, J. Yu, S. Zhang, A. D. Kent, and S. S. P. Parkin, Phys. Rev. Lett. 80, 5639 (1998).

${ }^{10}$ J. Yu, U. Ruediger, A. D. Kent, R. F. C. Farrow, R. F. Marks, D. Weller, L. Folks, and S. S. P. Parkin, J. Appl. Phys. 87, 6854 (2000).

${ }^{11}$ N. Garcia, M. Munoz, Y.-W. Zhao, Phys. Rev. Lett. 82, 2923 (1999).

${ }^{12}$ N. Garcia, M. Munoz, and Y.-W. Zhao, Appl. Phys. Lett. 76, 2586 (2000).

${ }^{13}$ I. A. Campbell and A. Fert, in Ferromagnetic Materials, edited by E. P. Wohlfarth (North-Holland, Amsterdam, 1992), Vol. 3.

${ }^{14}$ Ching-Ray Chang, IEEE Trans. Magn. 36, 1214 (2000).

${ }^{15}$ A. O. Adeyeye, G. Lauhoff, J. A. C. Bland, C. Daboo, D. G. Hasko, and H. Ahmed, Appl. Phys. Lett. 70, 1046 (1997).

${ }^{16}$ Th. G. S. M. Rijks, R. Coehoorn, M. J. M. de Jong, and W. J. M. de Jonge, Phys. Rev. B 51, 283 (1995). 